\title{
Hydrogen plasma treatments for passivation of amorphous-crystalline silicon-heterojunctions on surfaces promoting epitaxy
}

\author{
Mathias Mews, ${ }^{1}$, a) Tim F. Schulze, ${ }^{2}$ Nicola Mingirulli, ${ }^{1}$ and Lars Korte ${ }^{1}$ \\ ${ }^{1)}$ Helmholtz-Zentrum Berlin, Institute of Silicon Photovoltaics, Kekuléstraße 5, D-12489 Berlin, \\ Germany \\ ${ }^{2)}$ School of Chemistry, The University of Sydney, NSW 2006, Australia
}

(Dated: 26 September 2014)

The impact of post-deposition hydrogen plasma treatment (HPT) on passivation in amorphous/crystalline silicon (a-Si:H/c-Si) interfaces is investigated. Combining low temperature a-Si:H deposition and HPT, a high effective charge carrier lifetime $>8 \mathrm{~ms}$ is achieved on $\mathrm{c}-\mathrm{Si}<100>$, which serves as model for surfaces promoting epitaxy of a-Si:H. It is shown that the passivation improvement stems from diffusion of hydrogen atoms to the heterointerface and subsequent dangling bond passivation. Concomitantly, the a-Si:H hydrogen density increases, leading to band gap widening and void formation, while the film disorder is not increased. Thus, HPT allows for a-Si:H band gap and a-Si:H/c-Si band offset engineering.

Suppressing charge carrier recombination at surface dangling bonds on crystalline silicon ('passivation') is an important task in numerous semiconductor devices, including high-efficiency solar cells. Different materials, which provide passivation either by repelling charge carriers from the surface (field-effect passivation), or chemically saturate dangling bonds; have been developed. Undoped amorphous silicon [a-Si:H(i)] provides excellent chemical passivation and favorable properties as a heterojunction contact, which enables to build fullarea passivated hetero-p/n-junctions by deposition of doped/undoped a-Si:H stacks. This concept is the basis of the silicon heterojunction solar cell (SHJ-SC), which has been shown to reach conversion efficiencies $>23 \%^{1}$. The suppression of epitaxial growth during a-Si:H deposition by plasma-enhanced chemical vapor deposition (PECVD) is crucial for reaching high open-circuit voltage $\left(\mathrm{V}_{O C}\right)$ in SHJ-SCs, which can be challenging for $<100>$ oriented surfaces ${ }^{2}$, or in the presence of morphological features such as random pyramid textures ${ }^{3}$. In these cases, the a-Si:H deposition parameter range is restrained to regimes leading to inferior a-Si:H bulk quality - and thus passivation ${ }^{4}$ - by the requirement to suppress epitaxy. Recently it was shown that a HPT step during PECVD of a-Si:H/c-Si structures can lead to a passivation increase ${ }^{5}$.

Here we explore post-deposition hydrogen plasma treatment (HPT) as a means to improve passivation by a$\mathrm{Si}: \mathrm{H}(\mathrm{i})$ on surfaces promoting epitaxy, taking the example of the $\mathrm{Si}<100>$ surface which is technologically important for back-junction SHJ-SCs ${ }^{6}$. We analyze structural changes induced by HPT of ultra thin a-Si:H passivation layers, and relate them to the ensuing passivation improvement. Using a combination of Fourier-transform infrared spectroscopy (FTIRS), spectroscopic ellipsometry (SE), photoelectron spectroscopy (PES), and deuterium profiling, it is unequivocally shown that the pas-

\footnotetext{
a)mathias.mews@helmholtz-berlin.de
}

sivation improvement is caused by diffusion of atomic hydrogen $(\mathrm{H})$ towards the heterointerface. Concomitantly, the a-Si:H band gap $E_{\text {gap }}$ is widened by increased $\mathrm{H}$ incorporation, and the mass density reduced by the formation of microscopic voids. Contrary to previous reports ${ }^{5}$ the bond angle disorder in the a-Si:H network, for which the Urbach energy is a measure, is not increased by the HPT. Thus, post-deposition HPT enables passivation by a-Si:H(i) on surfaces promoting epitaxy and extend the usability of a-Si:H/c-Si heterojunctions to include thinfilm- or nanostructured silicon absorber materials, on which the affinity towards epitaxial growth prevents the preparation of a classical a-Si:H heteroemitter. Furthermore HPT induced changes in the band gap and band offsets offer additional degrees of freedom for tailoring the heterointerface.

In our study, we used $280 \mu \mathrm{m}$ thick $<100>$-oriented 1 $5 \Omega \mathrm{cm}$ phosphorus-doped high quality float zone silicon wafers as substrate material. Prior to deposition the wafers were cleaned following the RCA procedure and dipped in diluted hydrofluoric acid (2 min, 1\%) to strip off the native silicon oxide. a-Si:H(i)-layers were deposited with $\mathrm{PECVD}$ at $170^{\circ} \mathrm{C}$ using the 'LP' parameter set specified in Ref. 7. HPTs were done in a conventional parallel plate RF-PECVD $(13,56 \mathrm{MHz})$.The substrate temperature $\left(T_{\text {sub }}\right)$ was varied between 35 and $260{ }^{\circ} \mathrm{C}$. Layer thicknesses and band gaps of the a-Si:H layers were determined by SE on a Sentech SE850 (wavelength range $350-2500 \mathrm{~nm}$ ), followed by fitting of a TaucLorentz model ${ }^{8}$ to the data. Minority carrier lifetime $(\tau)$ measurements were conducted using a photoconductance decay (PCD) setup (Sinton Consulting WCT-100). $\mathrm{H}$ bonding configurations and total $\mathrm{H}$ content were quantified with FTIRS measured on a Bruker IFS 125HR in Brewster angle configuration ${ }^{9}$, and the calculation of the $\mathrm{H}$ content of the layers was done using the approach of Langford et $a l^{10}$. For characterization of the valence band tail, reflecting the bond angle disorder in the a-Si:H layers, near-ultraviolet PES (NUVPES) measurements were conducted in the constant-final-state-yield mode $(\mathrm{CFSYS})^{11}$. To prevent surface contamination after de- 


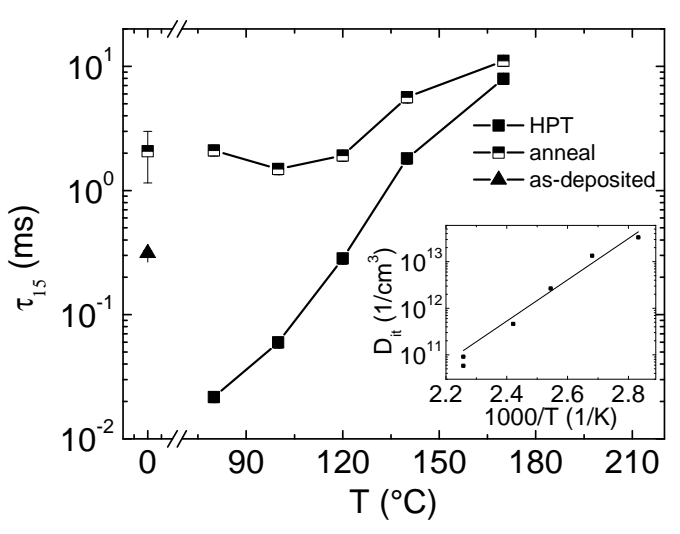

FIG. 1. Dependence of the free charge carrier lifetime $\tau_{15}$ at an injection level of $10^{15} \mathrm{~cm}^{-3}$ on the substrate temperature during a post deposition HPT. Filled symbols are values after HPT. Half-filled symbols mark the same samples after an additional thermal annealing. The triangle marks a sample that has not been exposed to a HPT. An Arrhenius-plot of the derived interface defect density $D_{i t}$ is displayed as inset.

position the samples have been vacuum transferred from the deposition chamber to the CFSYS analysis chamber. A model density of states (DOS) was fitted to the data to obtain the a-Si:H(i) parameters ${ }^{12}$.

We begin by analyzing the minority carrier lifetimes measured on c-Si samples symmetrically passivated with $7 \mathrm{~nm}$ of (i)a-Si:H, which were exposed to 4 min of HPT at different $T_{\text {sub }}$ after deposition, and subsequent anneal at $200^{\circ} \mathrm{C}$ for 20 min under ambient pressure and $\mathrm{N}_{2}$ flow. As seen in Fig. 1, the HPT leads to a dramatic improvement of carrier lifetime, with a monotonic increase of the passivation level with $T_{\text {sub }}$ (filled symbols). The lifetime of an annealed sample without HPT is placed at $T_{\text {sub }}=0^{\circ} \mathrm{C}$. For $T_{\text {sub }}>140^{\circ} \mathrm{C}$, HPT enables higher lifetimes than by thermal annealing alone. At $T_{\text {sub }}>170^{\circ} \mathrm{C}$ the lifetime reaches $8 \mathrm{~ms}$ even without thermal annealing, which is state-of-the-art passivation for thin (i)a-Si:H layers ${ }^{13-15}$. The distinct dependence of the passivation result on $T_{\text {sub }}$ lends itself to further analysis. The contribution of an eventual interface charge and the chemical passivation were disentangled using a semi-analytical model for interface recombination ${ }^{16}$, which was fitted to the measured PCD curves. In accordance with previous results on passivation by (i)a-Si:H, we found that the interface fixed charge is small $\left(Q_{\mathrm{f}}<-7 \times 10^{10} \mathrm{~cm}^{-2}\right)$, and the trend in $\tau$ is entirely dominated by the changes in the interface defect density $\left(D_{i t}\right)$. An Arrhenius plot of $D_{\text {it }}$ over $1 / T_{\text {sub }}$ (inset in Fig. 1) reveals an activation energy of $0.44 \mathrm{eV}$ for the defect saturation process upon HPT. This value is surprisingly close to the activation energy for in-diffusion of atomic $\mathrm{H}$ from a plasma into thick a-Si:H-films ${ }^{17}$. This suggests the diffusion of atomic $\mathrm{H}$ towards the heterointerface to be the underlying cause of the passivation effect observed in Fig. 1.
To corroborate this hypothesis, we performed deuterium (D) in-diffusion experiments on thin (i)a-Si:H passivation layers in order to correlate the deuterium profiles as measured by secondary-ion-mass spectroscopy (SIMS) with the ensuing passivation effect. To this aim, c-Si wafers were (i)a-Si:H coated on one side and treated with a deuterium-plasma (parameters identical to the HPT step used on all other samples). Afterwards the samples were coated with an additional a-Si:H capping layer at $T_{\text {sub }}=90^{\circ} \mathrm{C}$ to prevent oxidation of the deuterated layer and to provide a sacrifical layer for the initial sputtering phase of the SIMS measurement. The SIMS measurements were conducted at RTG Mikroanalyse $\mathrm{GmbH}$ with a Cameca IMS-4f using Argon ions at a kinetic energy of $6.5 \mathrm{keV}$.

$\mathrm{H}$ and $\mathrm{D}$ profiles of samples treated at different $T_{\text {sub }}$ with a D plasma are shown in Fig. 2. The a-Si:H(i)/c-Siinterface is at the origin of the abscissa and the shaded box marks the approximate position of the interface between the deuterated a-Si:H(i) and the capping layer. Both interfaces were assumed to coincide with the peaks in the oxygen profile (not shown). The $\mathrm{H}$ density in the (i)a-Si:H layer is diminished upon D in-diffusion, as would be expected based on previous results on thick aSi:H layers ${ }^{17}$. The D density in the a-Si:HD(i) increases with $T_{\text {sub }}$, as expected from the thermally activated indiffusion.

Furthermore the deuterium profiles show mostly linear decays, which implies that the amount of diffusing atoms is larger than the amount of trap states ${ }^{18}$. Additionally the profiles display $\mathrm{D}$ and $\mathrm{H}$ peaks close to the $\mathrm{a}-\mathrm{Si}: \mathrm{H} / \mathrm{c}-$ Si-interface, which is typical for epitaxy free a-Si:H/c-Siinterfaces ${ }^{19,20}$.

From Fig. 2b, it can be seen that resulting from the temperature dependence of the $\mathrm{D}$ diffusion coefficient, the $\mathrm{D}$ concentration at the heterointerface monotonically varies with the substrate temperature during $\mathrm{D}$ plasma treatment. Assuming that in-diffusion of atomic H/D promotes the defect saturation at the heterointerface and since $\mathrm{H}$ and $\mathrm{D}$ have the same diffusion coefficient ${ }^{17}$, it is tempting to relate the defect density as inferred from PCD measurements with the D concentration in the SIMS profiles. Fig. 2c shows the minority carrier lifetime of symmetrically passivated on both sides c-Si wafers (10 nm a-Si:H(i)) after a HPT, versus the D density at the a-Si:H(i)/c-Si-interface of the SIMS samples equivalently treated with a D plasma. Although the number of samples is small, a clear correlation is visible which underlines the role of atomic $\mathrm{H}$ diffusion towards the heterointerface in interface passivation.

Towards device application of HPT passivation layers, it is important to understand structural changes in the thin films upon HPT. In a previous report, based on the rather indirect measure of the broadening parameter $\mathrm{C}$ of the Tauc-Lorentz model for the a-Si:H dielectric function, it was invoked that the disorder in the amorphous network is increased by the HPT ${ }^{5}$. This would influence the stability of the a-Si:H passivation, as the disorder is 


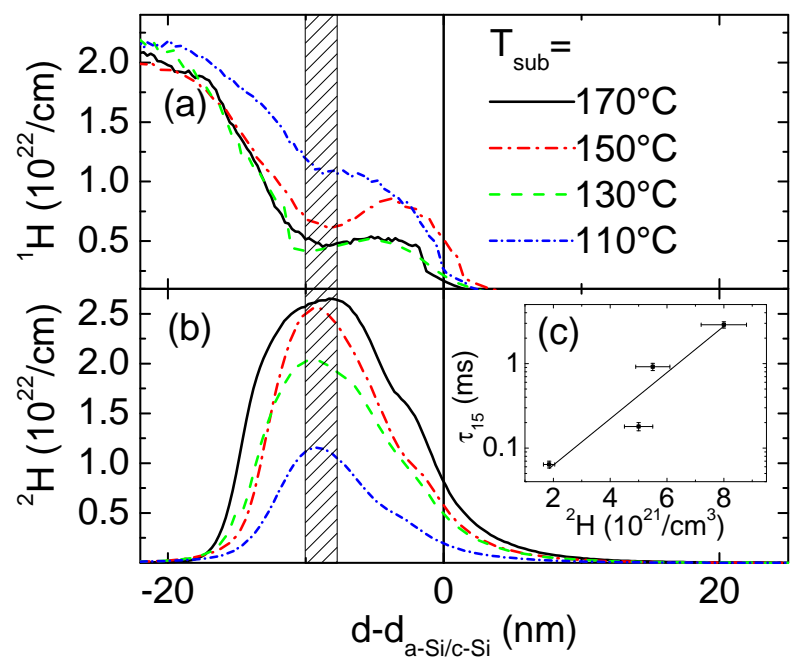

FIG. 2. (a) hydrogen $\left({ }^{1} \mathrm{H}\right)$ and (b) deuterium $\left({ }^{2} H\right)$ profiles of a-Si:H(i)/a-Si:DH(i)/c-Si-samples treated with a deuterium plasma at different $T_{\text {sub }}$. The single line shows the position of the a-Si:HD/c-Si-interface and the shaded box marks the approximate position of the a-Si:H/a-Si:HD-interface. (c) carrier lifetime in equivalently treated symmetrical samples with $10 \mathrm{~nm}$ a-Si:H(i) in dependence of the deuterium density at the interface.

an important parameter in the metastable defect creation processes in a-Si: $\mathrm{H}^{21}$.

In the following we analyze the $\mathrm{H}$ microstructure, mass density and valence band tail slope of the HPT treated a-Si:H layers in order to shed light on these suggested structural changes. Fig. 3 shows data on the $\mathrm{H}$ bonding, band gap and mass density, derived from SE and FTIRS measurements. The mass density is determined combining the hydrogen density (from FTIRS) and the longwavelength limit of the dielectric function (from SE) ${ }^{22}$. It is clearly visible from Fig. 3 that the overall $\mathrm{H}$ content increases along with the substrate temperature during the HPT, while at the same time the optical band gap is widened, and the mass density steeply decreases. Deconvolution of the $\mathrm{Si}-\mathrm{H}$ stretching modes $(\mathrm{SM})^{10}$ reveals that the low-frequency SM (LSM), usually associated with monohydrides, is decreased, while the high-frequency SM (HSM), commonly assigned to clustered monohydrides, is pronouncedly increased upon HPT. This implies that upon HPT there is a distinct reorganization of the $\mathrm{H}$ bonding configuration in the thin a-Si:H layer, and not just an increase of total Si-H bonds. In Fig. 3d the mass density is plotted versus the total $\mathrm{H}$ content. The slope of the mass density decay upon $\mathrm{H}$ incorporation is compatible with the formation of multi-vacancies like platelets or even (nanosized) voids in the a-Si:H film ${ }^{23}$. In summary, it is found that along with the well-known band gap widening, a loss of a-Si:H film compactness and a reorganization of $\mathrm{H}$ bonding in favor of multi-vacancyrelated bonding configuration is accompanying the HPT. A crucial question relates to the development of strain

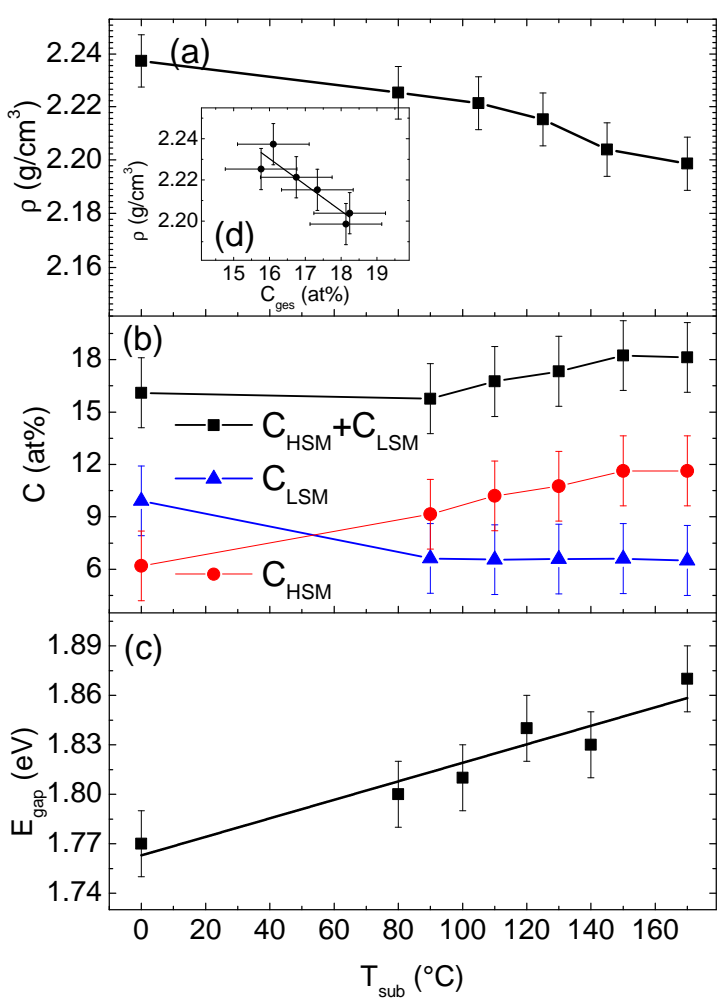

FIG. 3. (a) mass density, (b) hydrogen concentration and (c) band gap $\mathrm{E}_{\text {gap }} \rho$ of a-Si:H(i)-layers on c-Si substrates. In (b) black squares mark the total volume hydrogen content, red circles the HSM concentration and blue triangles the LSM concentration.

TABLE I. Parameters of the valence band edge before and after HPT, as determined by PES: Urbach energy $\left(E_{u}\right)$ and valence band edge $\left(E_{v}-E_{f}\right)$.

\begin{tabular}{cccc}
\hline sample hydrogen plasma & $E_{u}(\mathrm{meV})$ & $E_{v}-E_{f}(\mathrm{eV})$ \\
\hline A,B & no H-plasma & $63 \pm 5$ & $1.29 \pm 0.03$ \\
$\mathrm{C}$ & $60 \mathrm{MHz}$ & $70 \pm 5$ & $1.45 \pm 0.03$ \\
$\mathrm{D}$ & $13.56 \mathrm{MHz}$ & $70 \pm 5$ & $1.36 \pm 0.03$ \\
\hline
\end{tabular}

in the Si-Si network, being the source of metastable defect creation ${ }^{21}$. Therefore we analyzed the a-Si:H valence band tail, whose electronic DOS can be measured by PES. We conducted CFSYS measurements of four samples, two of which were exposed to a HPT at $13.56 \mathrm{MHz}$ and $60 \mathrm{MHz}$, respectively. The other two samples were references, which were deposited at identical conditions as the hydrogen plasma treated samples. The Urbach tail energies, characterizing the slope of the exponentially decaying valence band tail DOS, and the position of the valence band maximum relative to the Fermi level of these samples are compiled in Table I. From Table I it is obvious that the HPT shifts the valence band maximum away from the Fermi level by 70 to $150 \mathrm{meV}$, while the Urbach energy of the valence band tails is not af- 
fected. The first observation is consistent with the band gap widening found upon $\mathrm{HPT}^{5}$, which is known to proceed by a downward shift in energy of the valence band edge $^{24}$, and is also observed in our SE data (Fig. 3). The constant Urbach energy implies that there is no increase in the strain of the silicon network. This is interesting as the incorporation of higher $\mathrm{H}$ contents during PECVD growth usually leads to an increase of strain in a-Si: $\mathrm{H}^{24}$. However, in the present case, the post-deposition incorporation of hydrogen seems not to be related with an increase of strain. Interestingly, this finding is in accordance with previous studies on $\mathrm{H} / \mathrm{D}$ in-diffusion into thick a-Si:H films ${ }^{25,26}$. These authors observed clustering of excess hydrogen upon HPT, while no evidence of increased strain was found. Thus, it can be concluded that concerning structural changes upon HPT, ultrathin a-Si:H passivation layers behave similarly to thick a-Si:H specimen, i.e. display $\mathrm{H}$ clustering manifesting in an increase of the HSM and a loss of compactness along with band gap widening, while the level of strain remains constant. This renders the HPT particularly interesting for solar cell device applications for two reasons: Due to the constant and low Urbach energy hydrogen plasma treated films can be expected to be as stable as untreated ones against creation of metastable defects ${ }^{27}$ and the increase of the band gap with the hydrogen content is greater than for $\mathrm{H}$ rich layers directly deposited with PECVD, due to the missing compensating effect of a steeper band tail on the band gap widening ${ }^{24}$. This opens a new route towards band gap engineering, without severely deteriorating the electronic quality of the layers. Furthermore, the efficient in-diffusion of $\mathrm{H}$ from the plasma in combination with small thicknesses of the passivation layer allows for a dramatic increase of the passivation potential on c-Si surfaces. We achieved $\tau_{15}=11 \mathrm{~ms}$ and an implied $V_{\mathrm{OC}}$ of $737 \mathrm{mV}$ for a $\mathrm{HPT}$ at $170^{\circ} \mathrm{C}$ and thermal annealing of $7 \mathrm{~nm}$ a-Si:H. Finally, the possibility of deposition at suboptimal a-Si:H(i) to avoid epitaxy, combined with a post-deposition HPT, enables the application of heteroemitters on surfaces promoting epitaxy and (nano)structured surfaces. Thus, the two-step concept may be a feasible candidate for application of SHJ concepts to nanostructured absorbers, such as for nanowire $\operatorname{arrays}^{28}$.

In summary, a two-step approach towards highly passivating SHJ emitters, involving the deposition of a nonepitaxial a-Si:H(i)-layer and a HPT, has been investigated. The previously established improvement of the charge carrier lifetime at the a-Si:H(i)/c-Si-interface after a well-adjusted HPT was found to be due to diffusion of hydrogen atoms to the a-Si:H(i)/c-Si-interface and improved chemical passivation due to dangling bond saturation. Furthermore, this approach is highlighted to enable the preparation of state-of-the-art passivation layers on the silicon $<100>$-surface and surfaces which are prone to epitaxial growth, without introducing defect-creating strain into the amorphous network.

\section{ACKNOWLEDGMENTS}

The authors would like to thank Erhard Conrad, Thomas Lußky, Robert Rößler and Kerstin Jacob for experimental and technical assistance, and Karsten Holldack for assistance with FTIRS measurements. Financial support by the German Federal Ministry for Environment, Nature Conservation and Nuclear Safety (grant numbers 0325114A and 032511B) and by the Robert Bosch GmbH, Schott Solar AG, Sunways AG and the Stiebel Eltron GmbH \& Co. KG is acknowledged.

${ }^{1}$ T. Mishima, M. Taguchi, H. Sakata, and E. Maruyama, Sol. En. Mat. Sol. Cells 95, 18 (2011).

${ }^{2}$ U. K. Das, M. Z. Burrows, M. Lu, S. Bowden, and R. W. Birkmire, Appl. Phys. Lett. 92, 063504 (2008).

${ }^{3}$ S. Olibet, E. Vallat-Sauvain, L. Fesquet, C. Monachon, A. Hessler-Wyser, J. Damon-Lacoste, S. D. Wolf, and C. Ballif, phys. status solidi (a) 207, 651 (2010).

${ }^{4}$ H. Fujiwara and M. Kondo, Appl. Phys. Lett. 90, 013503 (2007).

${ }^{5}$ A. Descoeudres, L. Barraud, S. de Wolf, B. Strahm, D. Lachenal, C. Guérin, Z. C. Holman, F. Zicarelli, B. Demaurex, J. Seif, et al., Appl. Phys. Lett. 99, 123506 (2011).

${ }^{6}$ N. Mingirulli, J. Haschke, R. Gogolin, R. Ferré, T. F. Schulze, J. Düsterhöft, N.-P. Harder, L. Korte, R. Brendel, and B. Rech, Phys. Status Solidi - RRL 5, 159 (2011).

${ }^{7}$ T. F. Schulze, H. N. Beushausen, C. Leendertz, A. Dobrich, B. Rech, and L. Korte, Appl. Phys. Lett. 96, 252102 (2010).

${ }^{8}$ G. E. Jellison Jr. and F. A. Modine, Appl. Phys. Lett. 69, 371 (1996).

${ }^{9}$ M. Z. Burrows, U. K. Das, R. L. Opila, S. D. Wolf, and R. W. Birkmire, J. Vac. Sci. Technol. A 26, 683 (2008).

${ }^{10}$ A. A. Langford, M. L. Fleet, B. P. Nelson, W. A. Lanford, and N. Maley, Phys. Rev. B 45, 13367 (1992).

${ }^{11}$ M. Sebastiani, L. Di Gaspare, G. Capellini, C. Bittencourt, and F. Evangelisti, Phys. Rev. Lett. 75, 3352 (1995).

${ }^{12}$ L. Korte and M. Schmidt, J. Appl. Phys. 109, 063714 (2011).

${ }^{13}$ T. F. Schulze, H. N. Beushausen, T. Hansmann, L. Korte, and B. Rech, Appl. Phys. Lett. 95, 182108 (2009).

${ }^{14}$ J.-W. A. Schüttauf, K. H. M. van der Werf, I. M. Kielen, W. G. J. H. M. van Sark, J. K. Rath, and R. E. I. Schropp, Appl. Phys. Lett. 98, 153514 (2011).

${ }^{15}$ S. De Wolf, C. Ballif, and M. Kondo, Phys. Rev. B 85, 113302 (2012).

${ }^{16}$ C. Leendertz, N. Mingirulli, T. F. Schulze, J.-P. Kleider, B. Rech, and L. Korte, Appl. Phys. Lett. 98, 202108 (2011).

${ }^{17}$ P. V. Santos and W. B. Jackson, Phys. Rev. B 46, 4595 (1992).

${ }^{18}$ M. Kemp and H. M. Branz, Phys. Rev. B 47, 7067 (1993).

${ }^{19}$ H. Fujiwara, J. Koh, C. R. Wronski, and R. W. Collins, Appl. Phys. Lett. 70, 2150 (1997).

${ }^{20}$ T. Schulze, L. Korte, and B. Rech, Thin Solid Films 520, 4439 (2012).

${ }^{21}$ M. J. Powell and S. C. Deane, Phys. Rev. B 48, 10815 (1993).

${ }^{22}$ Z. Remes, M. Vanecek, P. Torres, U. Kroll, A. Mahan, and R. Crandall, J. Non-Cryst. Solids 227, 876 (1998).

${ }^{23}$ A. H. M. Smets and M. C. M. van de Sanden, Phys. Rev. B 76, 073202 (2007).

${ }^{24}$ T. F. Schulze, F. Ruske, B. Rech, and L. Korte, Phys. Rev. B 83, 165314 (2011).

${ }^{25}$ N. H. Nickel and W. B. Jackson, Phys. Rev. B 51, 4872 (1995).

${ }^{26}$ W. B. Jackson, P. V. Santos, and C. C. Tsai, Phys. Rev. B 47, 9993 (1993).

${ }^{27}$ H. Shirai, D. Das, J. ichi Hanna, and I. Shimizu, Appl. Phys. Lett. 59, 1096 (1991).

${ }^{28}$ S. W. Schmitt, F. Schechtel, D. Amkreutz, M. Bashouti, S. K. Srivastava, B. Hoffmann, C. Dieker, E. Spiecker, B. Rech, and S. H. Christiansen, Nano Lett. 12, 4050 (2012). 